\title{
Does CHA2DS2-VASc score predict mortality in chronic kidney disease?
}

\author{
Christos Goudis $^{1} \cdot$ Stylianos Daios ${ }^{1} \cdot$ Panagiotis Korantzopoulos $^{2} \cdot$ Tong Liu $^{3}$
}

Received: 29 March 2021 / Accepted: 17 June 2021 / Published online: 7 July 2021

(c) Società Italiana di Medicina Interna (SIMI) 2021

\begin{abstract}
Chronic kidney disease (CKD) is a leading cause of morbidity and mortality worldwide. Assessment of cardiovascular (CV) and all-cause mortality in CKD patients is of particular importance. CHA2DS2-VASc (congestive heart failure, hypertension, age $\geq 75$ years, diabetes, prior stroke, vascular disease, age 65-74 years, and sex) score was originally formulated to predict the annual thromboembolic risk in patients with nonvalvular atrial fibrillation (AF). The calculation of R2CHADS2 and R2CHA2DS2VASc scores awarded an additional 2 points for $\mathrm{CrCl}<60 \mathrm{~mL} / \mathrm{min}$ and $\mathrm{GFR}<60 \mathrm{~mL} / \mathrm{min} / 1.73 \mathrm{~m}^{2}$. Recent studies have investigated whether CHA2DS2-VASc and R2CHADS \pm VASC scores could be used to predict CV or all-cause mortality in patients with CKD. CHA2DS2-VASc score was proven to be a significant predictor of CV and all-cause mortality in CKD patients, and a higher CHA2DS2-VASc score was associated with increased mortality. These findings are quite promising, and they may help physicians to identify high-risk groups in this population.
\end{abstract}

Keywords CHA2DS2-VASc $\cdot$ Chronic kidney disease $\cdot$ Mortality

\section{Introduction}

\section{Chronic kidney disease: epidemiology and definition}

Chronic kidney disease (CKD) has been recognized as a leading cause of morbidity and mortality worldwide [1]. The global estimated prevalence of CKD is $13.4 \%$ [2] and has been almost doubled over the last three decades due to the decrease in mortality from cardiovascular (CV) and infectious diseases, as well as the population's progressive aging [1]. According to international guidelines, CKD is diagnosed if one or both of the following two criteria are met for at least 3 months: (a) glomerular filtration rate (GFR) $<60 \mathrm{~mL} / \mathrm{min}$ per $1.73 \mathrm{~m}^{2}$ (b) markers of kidney damage (1 or more): (i)

Christos Goudis

cgoudis@hotmail.com

1 Department of Cardiology, Serres General Hospital, 45110 Serres, Greece

2 First Department of Cardiology, University of Ioannina Medical School, Ioannina, Greece

3 Tianjin Key Laboratory of Ionic-Molecular Function of Cardiovascular Disease, Department of Cardiology, Tianjin Institute of Cardiology, Second Hospital of Tianjin Medical University, Tianjin 300211, People's Republic of China albuminuria [albumin to creatinine ratio (ACR) $\geq 30 \mathrm{mg} / \mathrm{g}$ ] (ii) urinary sediment abnormality (iii) electrolyte or other abnormality due to tubular disorder (iv) abnormalities on histology (v) structural abnormalities detected by imaging vi) history of kidney transplantation [3].

\section{Chronic kidney disease and mortality}

In 2016, CKD caused 1.19 million deaths globally, which has increased by $28.8 \%$ from 2006 [4]. According to World Health Organization estimates, CKD will rank 13th in 2030 among the most common causes of death [5]. The kidney early evaluation program (KEEP) enrolled patients at high risk for developing CKD and contributed to our understanding on $\mathrm{CV}$ risk stratification, prognosis and treatment in this setting [6]. CKD was proved to be a significant predictor (OR: 1.44 ; 95\% CI 1.27-1.63) of premature CV death, defined as the occurrence of myocardial infarction or stroke before 55 years in males and 65 years in females [6]. In a longitudinal analysis restricted to a subgroup of KEEP cohort with CKD, lower eGFR, increased albuminuria and diabetes mellitus (DM) have been found as significant predictors of mortality [7]. Both proteinuria and reduction in GFR can predict the development of fatal and non-fatal CV events, regardless of traditional $\mathrm{CV}$ risk factors, namely 
hypertension, smoking, hyperlipidemia, age and gender [8]. Therefore, assessing the risk of adverse outcomes and especially mortality associated with CKD is of particular importance for both physicians and patients [9]. At the moment, only one risk model has been developed to predict the occurrence of non-fatal $\mathrm{CV}$ events, renal failure requiring transplantation and death, in patients with eGFR $<30 \mathrm{ml} /$ $\mathrm{min} / 1.73 \mathrm{~m}^{2}$ based on parameters that are readily available in routine clinical practice (age, sex, race, eGFR, systolic blood pressure, history of $\mathrm{CV}$ disease, DM, urine ACR and smoking) [10].

\section{CHADS2, CHA2DS2-VASC, R2CHADS2 and R2CHA2DS2VASC scores}

CHADS2 is a simple score and was initially used for stroke risk stratification in AF. It was derived by the combination of stroke risk factors established in AFI and SPAF studies [11]. CHADS2 was formed by assigning 1 point each for the presence of congestive heart failure, hypertension, age $\geq 75$ years, DM and 2 points for history of stroke or transient ischemic attack [11]. CHADS2 manages well in identifying high-risk patients but provides ambiguous results in those at low or moderate stroke risk [12]. As a result, it was subjected to criticism for: (i) low discrimination ability for patients at low risk of stroke (ii) absence of important independent stroke and thromboembolic risk factors (iii) discrepancy between the original validation and further applications in guidelines and real-world cohorts [13, 14].

CHA2DS2-VASc [congestive heart failure, hypertension, age $\geq 75$ years, diabetes, prior stroke, vascular disease, age 65-74 years and sex (female) category] score was originally formulated to predict the annual thromboembolic risk in patients with nonvalvular AF $[15,16]$. CHA2DS2-VASc is a simple, effective and easy-to-use tool for truly low-risk and truly high-risk patients, but it has difficulties in tailoring anticoagulant treatment in AF patients at intermediate risk of stroke [17]. In individuals with AF and a CHA2DS2VASc score of 1, therapeutic decisions should be based on the individual balance between thromboembolic and bleeding risk [18].

R2CHADS2 score incorporates the components of CHADS2 score and awards 2 points for renal dysfunction $\left(\mathrm{CrCl}<60 \mathrm{~mL} / \mathrm{min}\right.$ and $\left.\mathrm{GFR}<60 \mathrm{~mL} / \mathrm{min} / 1.73 \mathrm{~m}^{2}\right)$ [19]. It was derived from study subjects enrolled in ROCKET-AF trial. The post hoc analysis of outcomes in ROCKET AF found that impaired renal function is independently associated with the occurrence of stroke or systemic embolism during follow-up of patients with a relatively high risk of stroke (mean CHADS2 score 3.5) receiving warfarin or rivaroxaban. R2CHADS2 score enhanced stroke risk assessment based on the net reclassification index by $8.2 \%$ compared with CHADS 2 score and by $6.2 \%$ compared with CHA2DS2-VASc score [19]. Piccini et al. applied this model to the ATRIA cohort of $>13000$ patients with nonvalvular AF to validate R2CHADS2 score in an independent population of patients with $\mathrm{AF}$ across a broader range of inherent risk [19]. R2CHADS2 risk score exhibited similar power to CHADS2 score regarding stroke occurrence (C statistics 0.672 and 0.673 , respectively), however, net stroke risk reclassification was improved $17.4 \%$ (95\% CI 12.1-22.5\%) with R2CHADS2 score. The findings were similar when applied to patients not receiving warfarin (C statistics 0.696 versus 0.704 for R2CHADS2 and CHADS2 scores, respectively). Net reclassification index improved with R2CHADS2 by $22.6 \%$ (95\% CI 14.5-30.7\%) [19].

In the same way, R2CHA2DS2VASc score awards an additional 2 points for $\mathrm{CrCl}<60 \mathrm{~mL} / \mathrm{min}$ and $\mathrm{GFR}<60 \mathrm{~mL} /$ $\min / 1.73 \mathrm{~m}^{2}$ and has already been used to predict long-term outcomes for patients with coronary artery disease (CAD)20 and acute coronary syndromes (ACS) [20-22].

\section{CHA2DS2-VASc score predicts mortality in several diseases}

The validity of CHA2DS2-VASc score in predicting ischemic stroke and thromboembolism has been extended beyond the originally proposed AF field. High CHA2DS2-VASc scores have been associated with increased mortality in patients with several diseases, irrespective of the presence or absence of AF. CHADS2, CHA2DS2-VASc and R2CHADS2 scores have been associated with higher incidence of mortality in patients with stable CAD and ACS [23]. Compared to CHADS2, CHA2DS2-VASc and R2CHADS2 scores provide better discrimination and reclassification for mortality. In addition, CHA2DS2-VASc and R2CHADS2 scores have comparable predictive ability of mortality to GRACE score [23]. CHA2DS2-VASc score was proven to be useful in predicting mortality in HF patients, irrespective of the presence or absence of AF, ischemic or non-ischemic etiology, and reduced or preserved EF [24]. In the same line, Chen et al. also reported that CHADS2, CHA2DS2-VASc and R2CHADS2 scores are moderately accurate predictors of all-cause mortality in systolic HF patients with or without AF. However, only CKD and R2CHADS2 scores seem to be independent predictors of 1 year all-cause mortality in this setting. In terms of predicting all-cause mortality in systolic HF patients, R2CHADS2 seems to be the best of the three scoring systems, especially in systolic HF patients without AF [25]. Moreover, CHA2DS2-VASC score highly predicts all-cause mortality in patients' acute ischemic stroke [26], ICD [27], and acute pulmonary embolism [28]. CHADS2, CHA2DS2-VASc and CHA2DS2-VASc-M (modified version of the CHA2DS2VASc scoring system in which 1 point was assigned to male 
instead of female sex) have also been significantly associated with all-cause mortality in COVID-19 patients [29]. The purpose of this paper is to present the latest knowledge and to describe recent studies investigating whether CHA2DS2-VASc score could be used to predict $\mathrm{CV}$ or all-cause mortality in CKD patients, with or without AF.

\section{CHA2DS2VASC and R2CHADS \pm VASC score in atrial fibrillation patients with chronic kidney disease}

In $\mathrm{AF}$ patients with end-stage renal disease (ESRD) on dialysis, Wang et al. reported that CHA2DS2-VASc score predicts mortality during follow-up [30]. Shih et al. also reported mortality in patients with new onset AF undergoing hemodialysis. An increase in CHA2DS2-VASc score was associated with increased mortality in this setting. The annual risk of all-cause death for patients with a CHA2DS2-VASc score of 0 was $10.03 \%$ and for those with a CHA2DS2-VASc score of 9 was $63.10 \%$, respectively [31]. Fu et al. refined the CHADS2 and CHA2DS$2 \mathrm{VASc}$ scores by combining creatinine clearance $(\mathrm{CrCl})$ and GFR, and evaluated the performance of $\mathrm{CrCl}$-based and GFR-based schemes in death risk stratification of Chinese patients $>60$ years old with AF. Renal function was evaluated with $\mathrm{CrCl}$ formula and different GFR formulas, as well. Five different kinds of R2CHADS2 and R2CHA2DS2VASc schemes were generated by combining $\mathrm{CrCl}$ and GFR with CHADS2 and CHA2DS2VASc scores [32]. Results provided evidence for the significantly better performance of GFR-based schemes-R2(GFR)CHADS2 and R2(GFR) CHA2DS2VASc - and the moderately better performance of $\mathrm{CrCl}$-based schemes- $\mathrm{R} 2(\mathrm{CrCl}) \mathrm{CHADS} 2$ and $\mathrm{R} 2(\mathrm{CrCl})$ CHA2DS2VASc - in death risk stratification compared with CHADS2 and CHA2DS2VASc scores [32]. These findings, however, were not confirmed in a recent study by Premuzic et al. The investigators sought to determine the association of eGFR with CV mortality in AF patients after 24 months of follow-up [33]. They concluded that CV mortality was independently associated with eGFR $(b=0.169$, $P=0.04)$, male gender $(b=0.156, P=0.03)$, CHA2DS2VASc $(b=0.467, P=0.02)$ and R2CHA2DS2VASc scores $(b=0.391, P=0.04)$ but not with R2CHADS2 score. In addition, R2CHA2DS2VASc was not associated with CV mortality more than CHA2DS2VASc [33].

\section{CHA2DS2VASC score in non-atrial fibrillation patients with chronic kidney disease}

Recent studies have also shown that CHA2DS2-VASc score can predict mortality in CKD patients. Pravda et al. investigated the association of CHA2DS2-VASc with mortality and major adverse $\mathrm{CV}$ outcomes in patients with ESRD on chronic hemodialysis [34]. A higher CHA2DS2-VASc score was associated with an increased risk for the composite endpoint of all-cause mortality, myocardial infarction, and stroke within the first year of hemodialysis in the low (CHA2DS2-VASc 0-3), intermediate (CHA2DS2-VASc 4-5) and high (CHA2DS2-VASc $\geq 6$ ) CHA2DS2-VASc groups, respectively $(P<0.01)$. A multivariate analysis using the CHA2DS2-VASc score group of $0-3$ as the reference group yielded adjusted ORs for the composite endpoint at 1 year that increased as the CHA2DS2-VASc score was higher $(\mathrm{OR}=2.695 \% \mathrm{CI} 1.6-4.2$ and $\mathrm{OR}=4.2,95 \%$ CI 3.3-7.5 for patients with the CHA2DS2-VASc score of $4-5$ and $\geq 6$, respectively, $P<0.01$ ) [34]. CHA2DS2-VASc score was also used as a continuous variable to assess the risk, and was shown that CHA2DS2-VASc score is associated with a $38 \%$ increased risk for the composite endpoint [34]. Hsu et al. evaluated the usefulness of CHADS2 and CHA2DS2-VASc scores for the prediction of CV and allcause mortality in CKD [35]. Age, male gender, hypertension, heart failure and CHA2DS2-VASc score (HR 1.600; 95\% CI 1.254-2.040; $P<0.001)$ were proven to be significant predictors of CV mortality. Similarly, age, male gender, heart failure, CHA2DS2-VASc score (HR 1.503; 95\% CI $1.300-1.739 ; P<0.001)$ and angiotensin II receptor blockers use were significant predictors of all-cause mortality. Higher stage of CKD was also associated with increased all-cause mortality in CKD [35]. Recently, Vodošek Hojs et al. performed a prospective study in non-dialysis CKD patients and assessed the role of CHA2DS2-VASc score in predicting CV and all-cause mortality in CKD. Kaplan-Meier survival analysis showed that $\mathrm{CV}(P=0.001)$ and allcause $(P=0.001)$ mortality were higher in patients with CHA2DS2-VASc score $>2$. CHA2DS2-VASc score was an independent predictor of CV (HR: 2.04, CI 1.20-3.45, $P=0.008$ ) and all-cause mortality (HR: 2.06, CI 1.43-2.97, $P=0.001$ ) [36]. The summarized studies investigating the ability of CHA2DS2-VASc score to predict CV and all-cause mortality in CKD are listed in Table 1.

\section{Conclusion}

CHA2DS2-VASc score has been widely used to evaluate the risk of stroke in AF patients [37], but has been shown to predict CV and all-cause mortality in CKD patients as well. Indeed, higher CHA2DS2-VASc scores have been associated with increased mortality. These findings are of particular importance because CKD is very common in clinical practice and utilization of CHA2DS2-VASc score may help physicians to identify high-risk groups in this setting. Intensive care along with modification of risk factors and treatment of coexisting diseases may improve prognosis in these patients. 
Table 1 Studies investigating the ability of CHA2DS2-VASc score to predict CV and all-cause mortality in chronic kidney disease

\begin{tabular}{lllll}
\hline Study (Reference) & AF cases /number of $\quad$ Age (years) $\quad$ Follow-up, years $\quad$ Database/type of study Key findings
\end{tabular}

Wang et al. [30]

Shih et al. [31]

$6.772 / 77.397$

301 AF patients

Pravda et al. [34]

$105 / 457$

Hsu et al. [35]

437 CKD patients

$68 \pm 12$ years

Fu et al. [32]

219 AF patients

Vodosek Hojs et al. [36]
70.6 years

2 years
91 months (25th- 75th percentile: 59-101)

86 years

1.11 years
$66 \pm 13 \quad 955 \pm 765$ days years

Retrospective observational
Retrospective observational

Retrospective cohort

Retrospective observational-longitudinal

Retrospective longitudinal

Retrospective observational

$60.3 \pm 12.8$ years $1696.5 \pm 564.6$ days Prospective
CHA2DS2-VASc detects mortality during follow-up $(c=0.638)$

The annual risk of all-cause mortality for patients with a CHA2DS2-VASc score of 0 was $10.03 \%$ and for those with a CHA2DS2-VASc score of 9 was $63.10 \%$, respectively

CV mortality was independently associated with CHA2DS2VASc $(b=0.467, P=0.02$ ) and R2CHA2DS2VASc scores $(b=0.391$, $P=0.04$ ) but not with R2CHADS2 score

CHA2DS2-VASc score predicted the primary composite endpoint (all-cause mortality, myocardial infarction, and stroke) with an AUC of 0.72 (95\% CI 0.69-0.75)

CHADS2 and CHA2DS2-VASc scores (both $P$ value $<0.001)$ were significant predictors of $\mathrm{CVr}$ and all-cause mortality in the multivariate analysis

C-statistics of GFRbased schemesR2(GFR)CHADS2 and R2(GFR)CHA2 DS2 VASc-significantly exceeded that of CHADS 2 and CHA2DS2VASc scores $(P<0.05$ for all $)$

CHA2DS2-VASc score was an independent predictor of allcause (HR: 2.06, CI 1.43-2.97, $P=0.001)$ and $\mathrm{CV}$ mortality(HR: 2.04, CI 1.20-3.45, $P=0.008$ )

$A F$ atrial fibrillation, $C K D$ chronic kidney disease 
More studies are needed to further confirm these promising findings so that CHA2DS2-VASc score can be widely used in clinical setting.

Funding None.

\section{Declarations}

Conflict of interest The author(s) declare that they have no conflict of interest.

Human and animal rights statement There is no research involving human participants and/or animals.

Informed consent There is no informed consent because there weren't any human participants.

\section{References}

1. Provenzano M, Coppolino G, Faga T et al (2019) Epidemiology of cardiovascular risk in chronic kidney disease patients: the real silent killer. Rev Cardiovasc Med 20:209-220. https://doi.org/10. 31083/j.rcm.2019.04.548

2. Lv JC, Zhang LX (2019) Prevalence and disease burden of chronic kidney disease. Adv Exp Med Biol 1165:3-15. https://doi.org/10. 1007/978-981-13-8871-2_1

3. Webster A, Nagler E, Morton R et al (2017) Chronic kidney disease. Lancet 389:1238-1252. https://doi.org/10.1016/S01406736(16)32064-5 (Epub 2016 Nov 23)

4. Ng JK, Li PK (2018) Chronic kidney disease epidemic: how do we deal with it? Nephrology (Carlton) 23(Suppl 4):116-120. https:// doi.org/10.1111/nep.13464

5. Mathers CD, Loncar D (2006) Projections of global mortality and burden of disease from 2002 to 2030. PLoS Med 3(1):e442

6. Whaley-Connell AT, Kurella Tamura M et al (2013) Advances in CKD detection and determination of prognosis: executive summary of the National Kidney Foundation-kidney early evaluation program (KEEP) 2012 annual data report. Am J Kidney Dis 61(4 Suppl 2):S1-3. https://doi.org/10.1053/j.ajkd.2013.01.006

7. McCullough PA, WhaleyConnell A, Brown WW et al (2010) Kidney early evaluation program (KEEP) investigators. Cardiovascular risk modification in participants with coronary disease screened by the kidney early evaluation program. Intern Med J 40:833-841

8. Matsushita K, van der Velde M, Astor BC et al (2010) Association of estimated glomerular filtration rate and albuminuria with allcause and cardiovascular mortality in general population cohorts: a collaborative meta-analysis. Lancet 375:2073-2081

9. Schneider MP, Eckardt KU (2019) Risk scores in patients with chronic kidney disease. Dtsch Med Wochenschr 144:739-742. https://doi.org/10.1055/a-0641-9625 (Epub 2019 Jun 4)

10. Grams ME, Sang Y, Ballew SH et al (2018) Predicting timing of clinical outcomes in patients with chronic kidney disease and severely decreased glomerular filtration rate. Kidney Int 93:14421451. https://doi.org/10.1016/j.kint.2018.01.009 (Epub 2018 Mar 29)

11. Gage BF, Waterman AD, Shannon W et al (2001) Validation of clinical classification schemes for predicting stroke: results from the National Registry of Atrial Fibrillation. JAMA 285:2864-2870
12. Nieuwlaat R, Capucci A, Lip GY et al (2006) Euro heart survey investigators antithrombotic treatment in real-life atrial fibrillation patients: a report from the Euro Heart Survey on atrial fibrillation. Eur Heart J 27:3018-3026

13. Karthikeyan G, Eikelboom JW (2010) The CHADS2 score for stroke risk stratification in atrial fibrillation-friend or foe? Thromb Haemost 104:45-48

14. Rietbrock S, Heeley E, Plumb J et al (2008) Chronic atrial fibrillation: incidence, prevalence, and prediction of stroke using the congestive HF, hypertension, age $\geq 75$, diabetes mellitus, and prior stroke or transient ischemic attack (CHADS2) risk stratification scheme. Am Heart J 156:57-64

15. Lip GYH, Nieuwlaat R, Pisters R et al (2010) Refining clinical risk stratification for predicting stroke and thromboembolism in atrial fibrillation using a novel risk factor-based approach: the Euro Heart Survey on atrial fibrillation. Chest 137:263-272

16. Camm AJ, Kirchhof P, Lip GYH et al (2010) Guidelines for the management of atrial fibrillation: the Task Force for the Management of Atrial Fibrillation of the European Society of Cardiology (ESC). Eur Heart J 31:2369-2429

17. van Doorn S, Debray TPA, Kaasenbrood F et al (2017) Predictive performance of the CHA2DS2-VASc rule in atrial fibrillation: a systematic review and meta-analysis. J Thromb Haemost 15:1065-1077. https://doi.org/10.1111/jth.13690 (Epub 2017 May 9)

18. Sulzgruber P, Wassmann S, Semb AG et al (2019) Oral anticoagulation in patients with non-valvular atrial fibrillation and a CHA2DS2-VASc score of 1: a current opinion of the European Society of Cardiology Working Group on Cardiovascular Pharmacotherapy and European Society of Cardiology Council on Stroke. Eur Heart J Cardiovasc Pharmacother 5:171-180. https://doi.org/ 10.1093/ehjcvp/pvz016

19. Piccini JP, Stevens SR, Chang Y et al (2013) Renal dysfunction as a predictor of stroke and systemic embolism in patients with nonvalvular atrial fibrillation: validation of the R(2)CHADS(2) index in the ROCKET AF (Rivaroxaban Once-daily, oral, direct factor Xa inhibition compared with vitamin $\mathrm{K}$ antagonism for prevention of stroke and Embolism Trial in Atrial Fibrillation) and ATRIA (AnTicoagulation and Risk factors In Atrial fibrillation) study cohorts. Circulation 127:224-232

20. Li Y, Wang J, Lv L et al (2018) Usefulness of the CHADS2 and R2CHADS2 scores for prognostic stratification in patients with coronary artery disease. Clin Interv Aging 13:565-571

21. Kiliszek M, Szpakowicz A, Filipiak KJ et al (2015) CHA2DS2VASc and R2CHA2DS2-VASc scores have predictive value in patients with acute coronary syndromes. Pol Arch Med Wewn 125:545-552

22. Wegiel M, Rakowski T, Dziewierz A et al (2018) CHA2DS2VASc and R2-CHA2DS2-VASc scores predict in-hospital and post-discharge outcome in patients with myocardial infarction. Adv Interv Cardiol 14:391-398

23. Huang FY, Huang BT, Pu XB et al (2017) CHADS2, CHA2DS2VASc and R2CHADS 2 scores predict mortality in patients with coronary artery disease. Intern Emerg Med 12:479-486. https:// doi.org/10.1007/s11739-017-1608-x (Epub 2017 Feb 4)

24. Chen YL, Cheng CL, Huang JL et al (2017) TSOC-HFrEF Registry investigators and committee. Mortality prediction using CHADS2/CHA2DS2-VASc/R2CHADS2 scores in systolic heart failure patients with or without atrial fibrillation. Medicine (Baltimore) 96:e8338. https://doi.org/10.1097/MD.0000000000008338

25. Yoshihisa A, Watanabe S, Kanno Y et al (2016) The CHA(2) DS(2)-VASc score as a predictor of high mortality in hospitalized heart failure patients. ESC Heart Fail 3:261-269. https://doi.org/ 10.1002/ehf2.12098 (Epub 2016 Jul 18)

26. Ntaios G, Lip GY, Makaritsis K et al (2013) CHADS2, CHA2S2DS2-VASc, and long-term stroke outcome in patients 
without atrial fibrillation. Neurology 80:1009-1017. https://doi. org/10.1212/WNL.0b013e318287281b (Epub 2013 Feb 13)

27. Hong C, Alluri K, Shariff N et al (2017) Usefulness of the CHA 2 DS 2-VASc score to predict mortality in defibrillator recipients. Am J Cardiol 120:83-86. https://doi.org/10.1016/j.amjcard.2017. 03.257 (Epub 2017 Apr 12)

28. Onuk T, Karataş MB, İpek $\mathrm{G}$ et al (2017) Higher CHA2DS2-VASc score is associated with increased mortality in acute pulmonary embolism. Clin Appl Thromb Hemost 23:631-637. https://doi. org/10.1177/1076029615627341 (Epub 2016 Jan 11)

29. Caro-Codón J, Lip GH, Rey JR et al (2021) Prediction of thromboembolic events and mortality by the CHADS2 and the CHA2DS2VASc in COVID-19. Europace. https://doi.org/10.1093/europace/ euab015

30. Wang TK, Sathananthan J, Marshall M et al (2016) Relationships between anticoagulation, risk scores and adverse outcomes in dialysis patients with atrial fibrillation. Heart Lung Circ 25:243-249. https://doi.org/10.1016/j.hlc.2015.08.012 (Epub 2015 Sep 25)

31. Shih CJ, Ou SM, Chao PW et al (2016) Risks of death and stroke in patients undergoing hemodialysis with new-onset atrial fibrillation: a competing-risk analysis of a nationwide cohort. Circulation 133:265-272. https://doi.org/10.1161/CIRCULATIONAHA.115. 018294 (Epub 2015 Dec 17)

32. Fu S, Zhou S, Luo L et al (2017) R2(GFR) CHADS 2 and R2(GFR) CHA2DS2VASc schemes improved the performance of CHADS2 and CHA2DS2VASc scores in death risk stratification of Chinese older patients with atrial fibrillation. Clin Interv Aging 2017(12):1233-1238. https://doi.org/10.2147/CIA.S1384 05.eCollection

33. Premužić V, Stevanović R, Radić P et al (2021) Chronic kidney disease and cardiovascular mortality in patients with atrial fibrillation: European Society of Hypertension project-ESH A Fib. Medicine (Baltimore) 100:e23975. https://doi.org/10.1097/ MD.0000000000023975

34. Pravda MS, Hagai KC, Topaz G et al (2020) Assessment of the CHA2DS2-VASc score in predicting mortality and adverse cardiovascular outcomes of patients on hemodialysis. Am J Nephrol 51:635-640. https://doi.org/10.1159/000508836 (Epub 2020 Jul 22)

35. Hsu PC, Lee WH, Chen SC et al (2020) Using CHADS(2) and CHA(2)DS(2)-VASc scores for mortality prediction in patients with chronic kidney disease. Sci Rep 10:18942. https://doi.org/ 10.1038/s41598-020-76098-y

36. Vodošek Hojs N, Ekart R, Bevc S et al (2021) CHA2DS2-VASc score as a predictor of cardiovascular and all-cause mortality in chronic kidney disease patients. Am J Nephrol. https://doi.org/10. $1159 / 000516121$

37. Hindricks G, Potpara T, Dagres N et al (2021) ESC Scientific Document Group. 2020 ESC Guidelines for the diagnosis and management of atrial fibrillation developed in collaboration with the European Association for Cardio-Thoracic Surgery (EACTS). Eur Heart J 42:373-498. https://doi.org/10.1093/eurheartj/ehaa6 12

Publisher's Note Springer Nature remains neutral with regard to jurisdictional claims in published maps and institutional affiliations. 